\title{
AN ADAPTIVE CONTROLLER FOR ENHANCING OPERATOR PERFORMANCE DURING TELEOPERATION
}

\section{Craig R. Carignan, Janice M. Tarrant}

ST Systems Corp.

Lanham, MD

\author{
Gary E. Mosier \\ Goddard Space Flight Center
}

Greenbelt, MD

\begin{abstract}
An adaptive controller is developed for adjusting robot arm parameters while manipulating payloads- of unknown mass and inertia. The controller is tested experimentally in a master/slave configuration where the adaptive slave arm is commanded via human operator inputs from a master. Kinematically similar six-joint master and slave arms are used with the last three joints locked for simplification. After a brief initial adaptation period for the unloaded arm, the slave arm retrieves different size payloads and maneuvers them about the workspace. Comparisons are then drawn with similar tasks where the adaptation is turned off. Several simplifications of the controller dynamics are also addressed and experimentally verified.
\end{abstract}

\subsection{INTRODUCTION}

Whether working in space, undersea, or on the ground, humans and robots must often contend with operating in an uncertain environment. One key characteristic often left unspecified is information about the object being manipulated. In the work presented here, a parameter identification scheme is used to estimate the parameters of the robot when maneuvering an unknown payload. The effect on performance of the improved model in tracking desired workspace trajectories is then examined.

The paper begins by giving the dynamics for the first three links of a parallel linkage robot arm. The parameters to be used in the identification algorithm are formulated from the link parameters. A parameter adaptive controller is developed for the slave arm which contains both a modeldependent feedforward term and a position/velocity feedback term. The master arm uses the same feedback as the slave arm with a reversal in sign. The algorithm is then tested experimentally on the teleoperated system doing payload retrieval tasks.

\subsection{DYNAMICS}

A set of minimaster hand controllers manufactured by Kraft Telerobotics Inc. was used in the teleoperation experiments: the left arm was used as the master and the right arm as the slave. Except for the rightheft symmetry, the two arms were identical. The Denavit-Hartenberg Parameters (see Craig [1]) for the first three links of the Kraft arm are given in Table 1.
Because of the parallel linkage used to drive the third joint (see Fig. 1), the Kraft arm dynamics could not be derived using recursive algorithms such as the Lagrangian Method or Newton-Euler Method (see Paul [2]). Instead, the method presented in Asada and Youcef-Toumi [3] was used, which is Lagrangian-based but does not assume open kinematic chains. Also pertinent to the development of the dynamics is the use of actuator coordinates and joint coordinates which need not be synonymous for parallel drive mechanisms. The reader is again referred to [3] for a discussion on this topic.

Following the procedure of Asada et al [3], the dynamics for the arm is derived in the form

$$
I=H \ddot{\theta}+C \dot{\theta}+I_{g}+D_{c} \operatorname{sgn}(\dot{\theta})+D_{\mu} \dot{\theta}
$$

The matrix $C$ is not unique and is chosen to be

$$
C_{i j}=\frac{1}{2} \sum_{k=1}^{N}\left(\frac{\partial H_{i j}}{\partial \theta_{k}}+\frac{\partial H_{i k}}{\partial \theta_{j}}-\frac{\partial H_{i k}}{\partial \theta_{i}}\right) \dot{\theta}_{k}
$$

because of its unique property of yielding the skew symmetric sum, $\dot{H}-2 C$. This is important in the formulation of the adaptive controller. $\mathrm{H}, \mathrm{C}$ and $I_{\mathrm{g}}$ for the Kraft arm are given in the appendix. $D_{c}$ and $D_{\mu}$ are diagonal matrices representing the magnitude of the coulomb friction and viscous friction coefficients, respectively.

Table 1: D-H Parameters for Kraft Minimaster.

\begin{tabular}{|c|c|c|c|}
\hline 1 & $a_{1-1}(\mathrm{~m})$ & $\alpha_{1}(\mathrm{deg})$ & $\alpha_{1}(\mathrm{~m}) *$ \\
\hline 1 & 0.000 & 0 & 0.000 \\
2 & 0.000 & -90 & \pm 0.075 \\
3 & $0.180\left(1_{2}\right)$ & 0 & \pm 0.055 \\
\hline
\end{tabular}

- the right arm has positive offsets in this coordinate convention and the left arm has negative offsets (note: $d_{2}=b_{1}$ and $d_{3}=b_{2}-b_{1}$ in Fig. 1).

\subsection{CONTROLLER}

The adaptive controller used in this study is the one developed by Slotine and Li [4]. As in the computed torque scheme, it contains feedforward terms to compensate for the

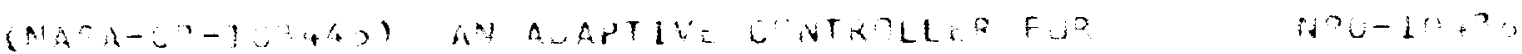

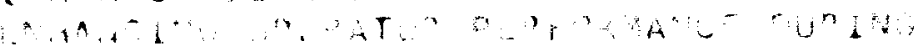

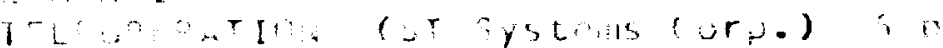

$$
\begin{aligned}
& \text { L UL I:I Un:I. }
\end{aligned}
$$




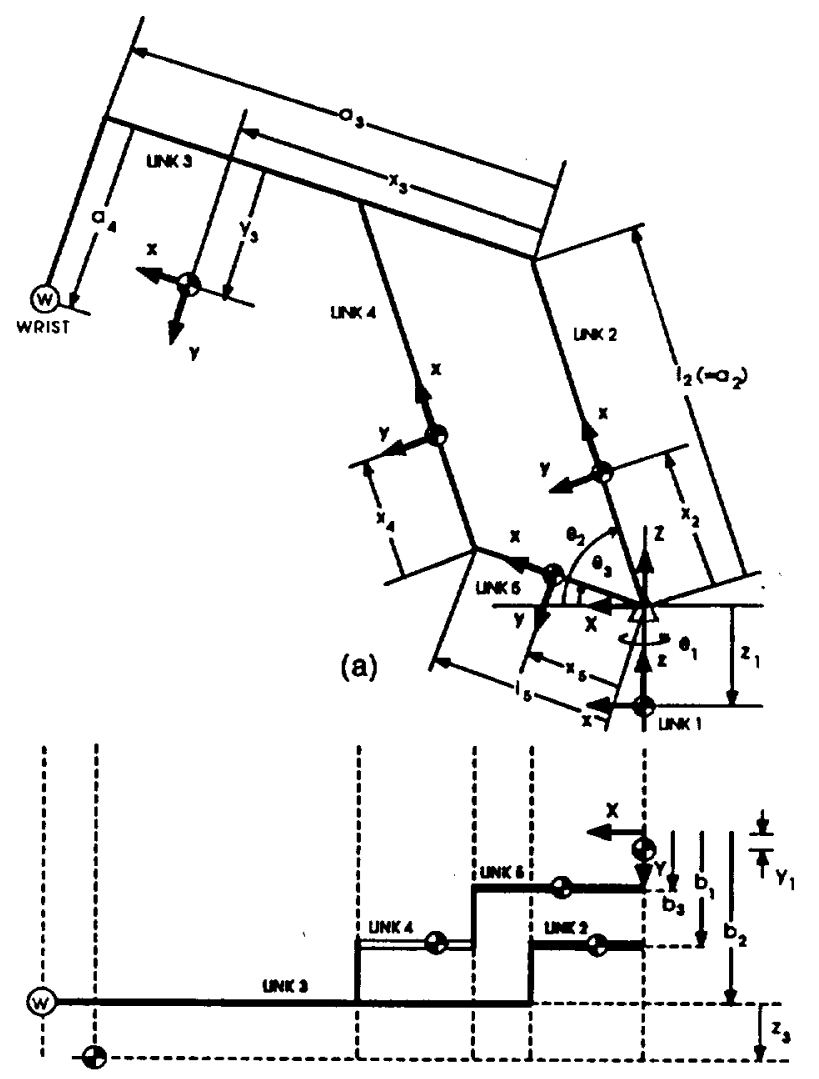

(b)

Figure 1: Kinematics for first three links of Kraft arm showing (a) side view and (b) top view.

dynamics, but it also has feedback terms to compensate for modeling uncertainty:

$$
\begin{aligned}
& I_{c o m}= \hat{H}(\theta) \ddot{\theta}_{r}+\hat{C}\left(\theta \cdot \dot{\theta} \dot{\theta}_{r}+\hat{I}_{g}(\theta)\right. \\
&+\hat{D}_{c} \operatorname{sgn}(\dot{\theta})+\hat{B}_{\mu} \dot{\theta}_{r}-K_{D s} \\
& \ddot{\theta}_{r}= \ddot{\theta}_{d}-\lambda \tilde{\theta} \\
& \dot{\theta}_{r}= \dot{\theta}_{d}-\lambda \tilde{\theta} \\
& \tilde{\hat{\theta}}= \theta-\theta_{d} \\
& \varepsilon=\tilde{\tilde{\theta}}+\lambda \tilde{\theta}
\end{aligned}
$$$$
\text { where } \quad \ddot{\theta}_{r}=\ddot{\theta}_{d}-\lambda \ddot{\theta}
$$

and $\theta_{d}(t)$ is the desired trajectory. The feedforward is adjustable through model updates, and the feedback, KDe. through trajectory updates if $K_{D}$ is constant. A block diagram depicting the control scheme is shown in Fig. 2.

The only requirement on $K_{D}$ is that it be positive definite. One desirable choice for $K_{D}$ is $\lambda \hat{A}$ (see Slotine and $L i$ [5]) which produces a feedback which depends on the model updates as well as the commanded trajectory. This yields a control law of the form

$$
\begin{aligned}
I_{c o m}= & \hat{H}(\theta)\left[\ddot{\theta}_{d}-2 \lambda \dot{\hat{\theta}}-\lambda 2 \tilde{\theta}\right]+\left(\hat{C}(\theta \cdot \dot{\theta})+\hat{D}_{\mu}\right)\left[\dot{\theta}_{d}-\lambda \tilde{\theta}\right] \\
& +\hat{I}_{g}(\theta)+\dot{D}_{c} \operatorname{sgn}(\dot{\theta})
\end{aligned}
$$

which is approximately the same as a computed torque with critically damped error dynamics.
The robot model, encompassed by the " $A$ " terms in (3), is linear in the model parameters $\hat{\underline{p}}$. $\hat{\mathrm{R}}$ is updated so as to ensure that the derivative of the Lyapunov function

$$
V(t)=\frac{1}{2} \mathbf{e}^{T} H_{e}+\frac{1}{2} \tilde{\mathbf{z}}^{\mathrm{T}} \Gamma \tilde{\mathrm{p}}
$$

is negative definite, where $\tilde{\mathrm{D}}=\hat{\mathrm{D}}-\mathrm{D}$, is the parametric error. The positive definite weighting matrix, $\Gamma$, is usually chosen to be diagonal. The parameter update law

$$
\dot{\mathrm{Q}}=-\Gamma^{-1} \mathrm{Y}^{\mathrm{T}} \mathrm{e}
$$

where

$$
\mathrm{Y} \tilde{\mathrm{p}}=(\hat{\mathrm{A}}-\mathrm{H}) \ddot{\boldsymbol{\theta}}_{\mathrm{r}}+(\hat{\mathrm{C}}-\mathrm{C}) \dot{\theta}_{r}+\left(\hat{\mathrm{I}}_{\mathrm{g}}-\boldsymbol{I}_{\mathrm{g}}\right)
$$

yields a Lyapunov derivative

$$
\dot{V}(t)=-e^{T} K_{D} \varepsilon
$$

thus ensuring stability for a positive definite choice of $\mathrm{K}_{\mathrm{D}}$. Because of their simpler form, the friction parameters have been omitted from (6) and (7) and are updated according to the analogous law

$$
\begin{aligned}
& \dot{\hat{\theta}}_{c_{k}}=-\gamma_{c k}^{-1} e_{k} \operatorname{sgn}\left(\dot{\theta}_{k}\right) \\
& \dot{B}_{\mu_{k}}=-\gamma_{\mu k}^{-1} e_{k} \dot{\theta}_{c k}
\end{aligned}
$$

where $k$ denotes the $k^{\text {th }}$ joint, and $D_{c_{k}}$ is the $(k, k)$ element of $D_{c}$. If $K_{D}$ is chosen to be $\lambda \hat{A}$, then $\ddot{\theta}_{r}$ must be replaced by $\ddot{\theta}_{r}-\lambda \mathrm{e}$ in (7) to account for the possibility that $\hat{A}$ is not positive definite. $\Gamma$ can be viewed as a gain which determines how quickly the parameters will adapt and is largely chosen heuristically.

Though this formulation ensures stability of the tracking algorithm for correct model structures, it says relatively little about the convergence of the model parameters; it does, however, guarantee convergence for "persistently exciting" trajectories. What this terminology means in the nonlinear case is an area of ongoing research, although in an intuitive sense, it means the trajectory must excite the dynamics in such a way that incorrect parameters will produce significant differences between the modeled and observed dynamics.

In the linear case, persistent excitation depends on the number of input frequencies used and how that relates to the order of the system. However, in tests where the number of input frequencies were varied, the convergence of the model parameters did not appear to be significantly effected in a one minute trial. It is possible that the speed of convergence depends more on other factors such as the number of parameters than on the degree of persistent excitation.

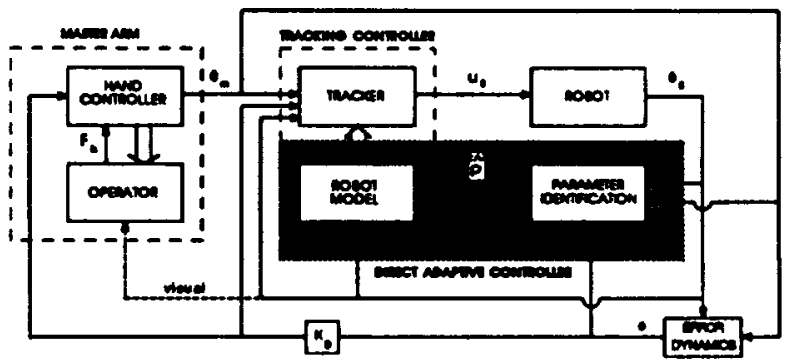

Fipure 2: 8chemattc of masker/elave visoperator wh direct adaptive controlier. 
Asymptotic convergence is only guaranteed when the parameters appear explicitly in the Lyapunov derivative. In "composite" control formulations, both state feedback and torque feedback are used and the model parameters appear explicitly in $\dot{V}(t)$, However, improved convergence was not apparent for this scenario and was computationally expensive; thus the experiments performed here used the simpler, noncomposite form of control. For more on this topic, the reader is referred to Slotine and $\mathrm{Li}[6]$.

\subsection{EXPERIMENTS}

A photograph of the Kraft "slave" arm used in the teleoperation tasks is shown in Figure 3. Both arms are clamped at the wrist so that only the first three degrees of freedom in the base are active. A hook was attached to the clamp on the right arm enabling it to retrieve weights during the tests. The AC motors were driven by control units that were supplied by the arm manufacturer.

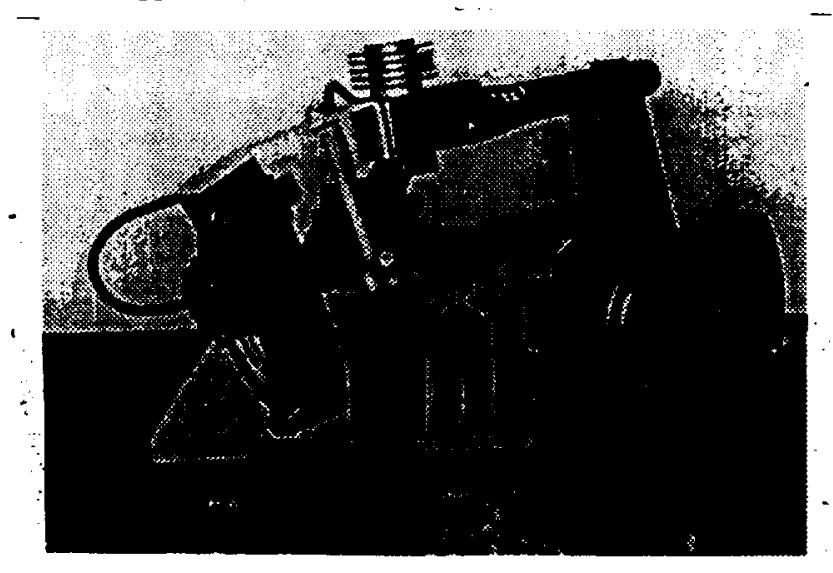

Figure 3: Photo of Kraft arm manipulatiag payload.

The only sensor information available was the position of the links supplied by potentiometers geared to the motor shafts. Velocity and acceleration information were obtained through differentiation and extensive filtering of the potentiometer data. A Compaq 386/20 equipped with coprocessor was used to derive the control laws and transfer data back and forth to the control unit. The experiments were run at $81 \mathrm{~Hz}$, the maximum rate achievable with the current software. The control bandwidth, $\lambda$, was chosen to be $1 / 2 \mathrm{~Hz}$, just slightly below the natural frequencies of the drive systems.

In all tests, the subjects were asked to move the arm to pick up the weight from a table, move the arm back to its initial position, sequentially move the shoulder aximuth $\left(\theta_{1}\right)$, shoulder elevation $\left(\theta_{2}\right)$, and elbow $\left(\theta_{3}\right)$ back and forth several times, and then to return the weight to the table. For each weight tested, the controller was first tried with the adaptation turned on and then turned off. Prior to running all the tests, one adaptive nun was made without a payload to obtain initial values for the arm parameters. These values were used in all subsequent runs as the starting parameters.

The master and slave joint positions for one set of tests run with the $0.5 \mathrm{~kg}$ weight are plotted in Figs. 4 and 5 . For the adaptive results shown in Fig. 4, the master and slave angles were nearly indistinguishable. In the nonadaptive plots, however, the master and slave shoulder elevations and elbow angles differed a great deal. This is because of the increasing effect of gravity on the loaded arm as one progresses toward the payload. Since the gravity parameters are nonadaptive and the gravity forces dominate the inertial forces for moderate speeds, joint 3 differed greatly from the commanded angle, whereas joint 1 , whose axis was parallel to gravity, was relatively unaffected.

It is almost possible to track the progress of the experiment simply by looking at Fig. 5: at $7 \mathrm{sec}$, the slave arm reaches the payload and begins pushing down on it (slave arm is impeded by the object so that $\theta_{3}$ for the slave < $\theta_{3}$ of the master -- pitch down produces positive $\theta_{3}$ ); at 17 $\mathrm{sec}$, the arm starts lifting the payload and at $20 \mathrm{sec}$, the payload clears the table (the slave cannot lift the payload as high as the master arm so that now $\theta_{3}$ for the slave $>\theta_{3}$ of the master). Beginning at 23,35 , and $45 \mathrm{sec}$, respectively, joints 1,2 , and 3 are moved back and forth, and at $57 \mathrm{sec}$, the payload is returned to the table.

Although the joint angle errors provide a fairly good measure of the tracking performance, the tracking error provides the true measure of the control system performance. Figure 6 plots the tracking errors for these two tests on the same graphs. These plots reaffirm the conclusions reached earlier from observations of the joint angles. In the adaptive case, it is difficult to discern when the payload is picked up, whereas for the nonadaptive case, this is relatively easy: at $t=7 \mathrm{sec}, s_{3}$ begins rising rapidly, and at $t=17 \mathrm{sec}, \mathrm{s}_{2}$ also begins to rise as the payload is lifted from the table.

The same experiments were repeated for retrieving a $1 \mathrm{~kg}$ weight, and results for joint 3 in the adaptive case are shown in Fig. 7. Even with a payload twice as large, the tracking errors are not significantly different from those of the $0.5 \mathrm{~kg}$ case in Fig. 6. This illustrates the ability of the tracker to maintain control performance when the arm's characteristics are being significantly altered through interaction with the environment.

As a final test, several adaptive runs were made with structural simplifications in the model used by the controller. Figure 8 shows joint 3 results for a trial in which the velocity dependent terms, i.e. $\hat{C} \dot{\theta}, \hat{B}_{c}$, and $\hat{B}_{\mu}$, were ignored. In comparing Fig. 8 with $s_{3}$ in Fig. 6, one can discern little degradation in going to the less accurate model. This is probably due to one of two reasons: the tracker was able to compensate for this inaccuracy through the adaptation of other parameters (for the friction parameters) or further modification of already existent parameters (for the Coriolis terms), or the neglected terms were insignificant for the particular tests which were run. The latter case seems unlikely for the friction parameters at least, since the coulomb friction forces were found to account for about $20 \%$ of the peak torque. Whatever the case, ignoring these terms can significantly reduce the amount of computation and is a possibility which should not be overlooked for more complex systems.

\subsection{CONCLUSIONS}

This effort applied a parameter adaptive controller to a parallel linkage arm and tested the controller on a teleoperated system. One purpose of this task was to verify 

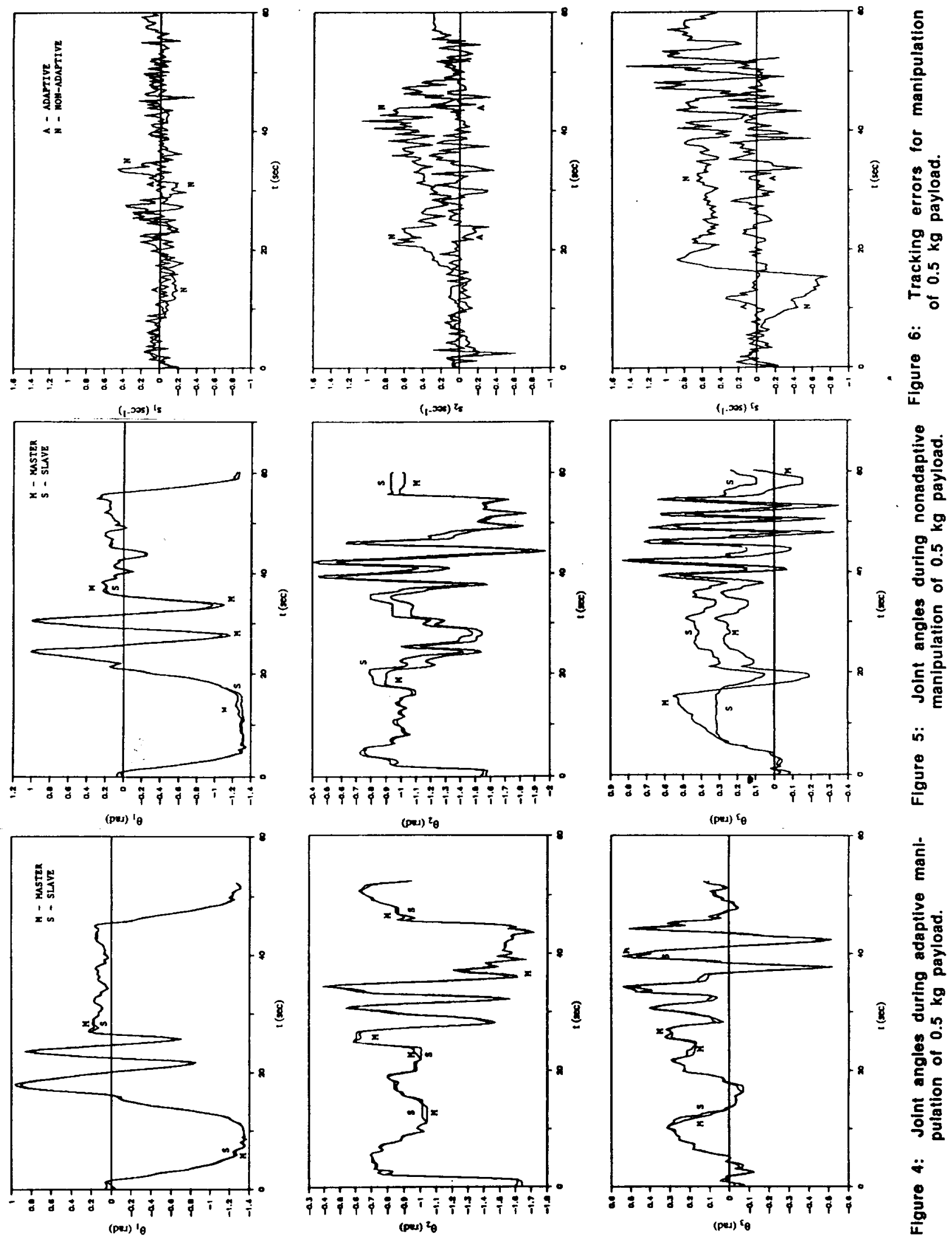
that the adaptation algorithm would work even if the commanded inputs were provided by a human operator. Another was to investigate the differences, in terms of improved manipulation ability, between a controller with invariant characteristics and one which accomodates extemal perturbations.

In experimental tests, the adaptive tracking algorithm was found to converge within several seconds following picking up or putting down a payload. This was an improvement over the nonadaptive controller where the errors built up considerably because of the inability of the feedforward to correctly compensate for the added gravitational forces on the arm due to picking up a payload. While this limitation did not unduly impede the operator in these tests, a more precise task would force the operator into moving the master arm along a "false" trajectory to achieve the desired motion in the slave arm. In a scenario dominated by inertial rather than gravitational forees, this inability would be manifested as large overshoots for the slave arm. In either situation, the range of motion for the slave arm may become severely restricted compared to the master.

The only way to reduce the error without modifying the feedforward in the nonadaptive case would be to increase the feedback gains on the position and velocity errors. These gains can only be increased to a certain extent, however, before the natural frequencies of the arm are excited $(\sim 1 \mathrm{~Hz})$. By contrast, the adaptive controller is able to maintain the same errors before and after a payload is picked up without changing the bandwidth.

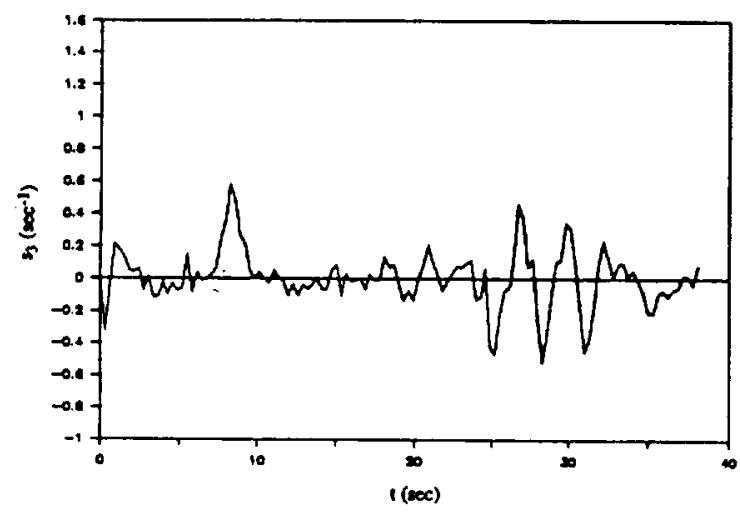

Figure 7: Joint 3 tracking error during adaptive manlpulation of $1.0 \mathrm{~kg}$ payload.

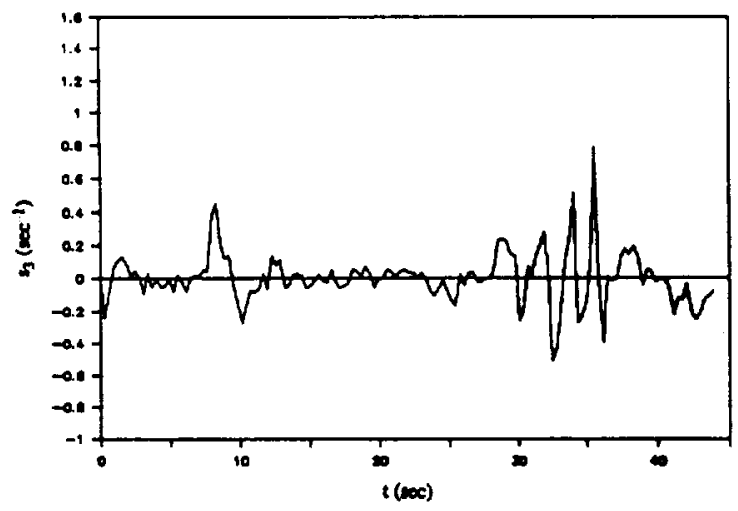

Figure s: Joint 3 trackine error ouring edeptive maniputation of $0.5 \mathrm{~kg}$ payload without velocity feedtionward.
Because the gravity forces dominated the arm torques in these experiments, the desired trajectory played only a minor role in the parameter adaptation. This is born out by observing that the joint 1 errors for the adaptive case decreased little if any over those for the nonadaptive case. (Joint 1 torques had no direct dependence on gravity.) For the trajectory to play a more important role, the inertial forces would have to be much higher relative to gravity. Thus it is not possible to extend all the conclusions drawn here on tracking convergence to a gravity-free environment.

One drawback worth mentioning in using this approach for teleoperation occurs during contact of the robot arm with immobile objects. When the progress of the robot arm is impeded by an object such as a table surface, the adaptive controller begins modifying the arm parameters to reflect an increase in the mass of the arm which could account for the inability of the arm to continue along its path. As the arm is commanded away from the surface, the movement is delayed as the arm parameters readjust to the newly found freedom of motion. If the exact position and orientation of the surface were known (such as in a completely automated setting), then the arm-environment interaction could be modeled and the contact could be taken into account in the tracking errors and the false modification of the arm parameters would not take place (see Niemeyer and Slotine [7]). Thus during compliant motion, the adaptation should be turned off to prevent their adjustment during this interaction.

\section{ACKNOWLEDGMENTS}

The authors would like to express their appreciation to the Space Station Project Office (Code 400.6), NASA Goddard Space Flight Center for their support of this research. Thanks also go to Rick Schnurr (Code 712.3) for providing the communications software for the Krafu/Compaq interface.

\section{REFERENCES}

1. Craig, J. J., Introduction to Bobotics, Addison-Wesley, Menlo Park, Calif., (O) 1986

2. Paul, R. P., Robot Manipulators: Mathematics. Programming, and Control, MIT Press, Cambridge, Mass., (C) 1981

3. Asada, H., and Youcef-Toumi, K., Direct-Drive Robots, MIT Press, Cambridge, Mass., O 1987

4. Slotine, J-J. E., and Li, W., "On the Adaptive Control of Robot Manipulators," Int. J. Robotics Research, Vol. 6, No. 3, 1987

5. Slotine, J-J. E., and Li, W., "Adaptive Manipulator Control: A Case Study," IEEE Trans. on Automatic Control, Vol. 33, No. 11, 1988

6. Slotine, J-J. E., and Li, W., "Composite Adaptive Control of Robot Manipulators," Automatica, Vol. 25, No. 4, 1989

7. Niemeyer, G., and Slotine, J-J. E., "Computational Algorithms for Adaptive Compliant Motion," Proc. IEEE Conf. on Robotics and Automation, Scottsdale, Ariz., May 1989 


\section{APPENDIX: KRAFT ARM DYNAMICS}

$I=H \ddot{\theta}+C \dot{\theta}+I_{g}$

where

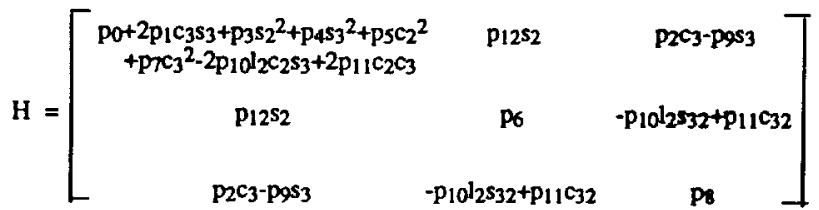

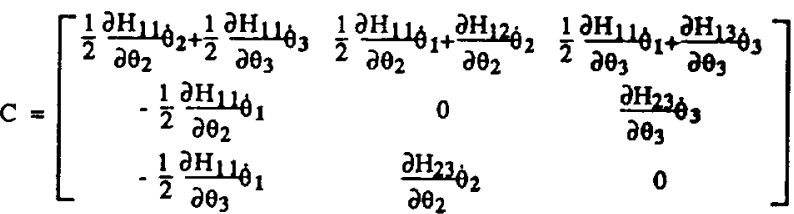

$I_{g}=\left[\begin{array}{c}0 \\ -p_{13} c_{2} \\ p_{10} s_{3}-p_{14} c_{3}\end{array}\right] g$

$\frac{\partial H_{11}}{\partial \theta_{2}}=2\left(p_{3}-p_{5}\right) s_{2} c_{2}+2 p_{10} l_{2} s_{2} s_{3}-2 p_{11} s_{2} c_{3}$

$\frac{\partial \mathrm{H}_{11}}{\partial \theta_{3}}=2\left(\mathrm{p}_{4}-\mathrm{p}_{7}\right) \mathrm{s}_{3} \mathrm{c}_{3}+2 \mathrm{p}_{1} \mathrm{c}_{3}^{2}-2 \mathrm{p}_{1} \mathrm{~s}_{3}^{2}-2 \mathrm{p}_{10} \mathrm{l}_{2} \mathrm{c}_{2} \mathrm{c}_{3}-2 \mathrm{p}_{11} \mathrm{c}_{2} \mathrm{~s}_{3}$

$\frac{\partial \mathrm{H}_{23}}{\partial \theta_{2}}=\mathrm{p}_{10} \mathrm{l}_{2} \mathrm{c}_{32}+\mathrm{p}_{11} \mathrm{~s}_{32}$

$\frac{\partial \mathrm{H}_{12}}{\partial \theta_{2}}=\mathrm{p}_{12} \mathrm{c}_{2}$

$\frac{\partial \mathrm{H}_{13}}{\partial \theta_{3}}=-\mathrm{p}_{2} \mathrm{~s}_{3}-\mathrm{pgc}_{3}$

$\frac{\partial \mathrm{H}_{23}}{\partial \theta_{3}}=-\mathrm{p}_{10} \mathrm{l}_{2} \mathrm{c}_{32}-\mathrm{p}_{11} \mathrm{~s}_{32}$

model parameters:

$\begin{array}{ll}p_{0}=I_{1 z} & p_{8}=I_{35 z z}+m_{4} I_{5}^{2} \\ p_{1}=I_{3 x y} & p_{9}=I_{3 x z}-m_{4} l_{5} b_{1}-m_{5} x_{5} b_{3} \\ p_{2}=I_{3 y z} & p_{10}=m_{3} y_{3} \\ p_{3}=I_{24 x x} & p_{11}=m_{3} x_{3} l_{2}+m_{4} x_{4} l_{5} \\ p_{4}=I_{35 x x} & p_{12}=m_{2} x_{2} b_{1}+m_{4} x_{4} b_{1}+m_{3} l_{2}\left(b_{2}+z_{3}\right) \\ p_{5}=I_{24 y y}+m_{3} l_{2}^{2} & p_{13}=m_{2} x_{2}+m_{3} l_{2}+m_{4} x_{4} \\ p_{6}=I_{24 z z}+m_{3} l_{2}^{2} & p_{14}=m_{3} x_{3}+m_{4} l_{5}+m_{5} x_{5} \\ p_{7}=I_{35 y}+m_{4} l_{5}^{2} & \end{array}$

${ }^{*} s_{j}=\sin \theta_{i}$ and $c_{i}=\cos \theta_{i}, s 32=\sin \left(\theta_{3}-\theta_{2}\right)$ and $c_{32}=\cos \left(\theta_{3}-\theta_{2}\right)$
The capital "I" 's are combinations of link inertias and mass $x \mathrm{CM}^{2}$ terms:

$$
\begin{array}{ll}
I_{1 z z}=i_{1 z z}+m_{1} y_{1}^{2} & I_{2 x x}=i_{2 x x}+m_{2} b_{1}^{2} \\
I_{2 z z}=i_{2 z z}+m_{2} x_{2}^{2} & I_{2 y y}=i_{2 y y}+m_{2} x_{2}^{2}+m_{2} b_{1}^{2} \\
I_{3 x x}=i_{3 x x}+m_{3} y_{3}^{2}+m_{3}\left(z_{3}+b_{2}\right)^{2} & I_{3 x y}=i_{3 x y}-m_{3} x_{3} y_{3} \\
I_{3 y y}=i_{3 y y}+m_{3} x_{3}^{2}+m_{3}\left(z_{3}+b_{2}\right)^{2} & I_{3 x z}=i_{3 x z}-m_{3} x_{3}\left(z_{3}+b_{2}\right) \\
I_{3 z x}=i_{3 z z}+m_{3} x_{3}^{2}+m_{3} y_{3}^{2} & I_{3 y z}=i_{3 y z}+m_{3} y_{3}\left(z_{3}+b_{2}\right) \\
I_{4 x x}=i_{4 x x}+m_{4} b_{1}^{2} & I_{5 x x}=i_{5 x x}+m_{5} b_{3}^{2} \\
I_{4 y y}=i_{4 y y}+m_{4} x_{4}^{2}+m_{4} b_{1}^{2} & I_{5 y y}=i_{5 y y}+m_{5} x_{5}^{2}+m_{5} b_{3}^{2} \\
I_{4 z z}=i_{4 z z}+m_{4} x_{4}^{2} & \\
I_{35 x x}=I_{3 x x}+I_{5 x x} & I_{5 z z}=i_{5 z z}+m_{5} x_{5}^{2} \\
I_{35 y y}=I_{3 y y}+I_{5 y y} & I_{24 x x}=I_{2 x x}+I_{4 x x} \\
I_{35 z z}=I_{3 z z}+I_{5 z z} & I_{24 y y}=I_{2 y y}+I_{4 y y} \\
& I_{24 z z}=I_{2 z z}+I_{4 z z}
\end{array}
$$

\begin{tabular}{|c|c|c|}
\hline $\begin{array}{l}p_{0}=0.0489 \\
p_{1}=0.0021 \\
p_{2}=-0.0214 \\
p_{3}=0.0521 \\
p_{4}=0.0807\end{array}$ & $\begin{array}{l}\mathrm{p}_{5}=0.0973 \\
\mathrm{P}_{6}=0.3449 \\
\mathrm{P}_{7}=0.3999 \\
\mathrm{p}_{8}=0.5819 \\
\mathrm{p}_{9}=-0.0209\end{array}$ & $\begin{array}{l}p_{10}=0.0523 \\
p_{11}=0.0827 \\
p_{12}=0.0088 \\
p_{13}=0.0650 \\
p_{14}=0.0996\end{array}$ \\
\hline
\end{tabular}

The $\mathrm{CM}$ positions in the local coordinate frames are:

$$
\begin{aligned}
& x_{\mathrm{cm} 1}=\left[\begin{array}{l}
0 \\
y_{1} \\
z_{1}
\end{array}\right], x_{\mathrm{cm} 2}=\left[\begin{array}{c}
x_{2} \\
0 \\
0
\end{array}\right], x_{\mathrm{cm} 3}=\left[\begin{array}{l}
x_{3} \\
y_{3} \\
z_{3}
\end{array}\right], \\
& x_{\mathrm{cm} 4}=\left[\begin{array}{c}
x_{4} \\
0 \\
0
\end{array}\right], x_{\mathrm{cm} 5}=\left[\begin{array}{c}
x_{5} \\
0 \\
0
\end{array}\right]
\end{aligned}
$$

The link inertia tensors about the $\mathrm{CM}$ in the local coordinate frames are:

$$
\begin{aligned}
& I_{1}^{1}=\left[\begin{array}{ccc}
i_{1 x x} & 0 & 0 \\
0 & i_{1 y y} & -i_{1 y z} \\
0 & -i_{1 y z} & i_{1 z z}
\end{array}\right], I_{2}^{2}=\left[\begin{array}{ccc}
i_{2 x x} & 0 & 0 \\
0 & i_{2 y y} & 0 \\
0 & 0 & i_{2 z z}
\end{array}\right], \\
& I_{3}^{3}=\left[\begin{array}{ccc}
i_{3 x x} & -i_{3 x y} & -i_{3 x z} \\
-i_{3 x y} & i_{3 y y} & -i_{3 y z} \\
-i_{3 x z} & -i_{3 y z} & i_{3 z z}
\end{array}\right], I_{4}^{4}=\left[\begin{array}{ccc}
i_{4 x x} & 0 & 0 \\
0 & i_{4 y y} & 0 \\
0 & 0 & i_{4 z z}
\end{array}\right], \\
& I_{5}^{5}=\left[\begin{array}{ccc}
i_{5 x x} & 0 & 0 \\
0 & i_{5 y y} & 0 \\
0 & 0 & i_{5 z z}
\end{array}\right]
\end{aligned}
$$

where the elements of $I$ are moments of inertia about various axes, e.g. $i_{x x}=\iint\left(y^{2}+z^{2}\right) d m$ and $i_{x y}=\iint x y d m$.

The initial parameter values used in all the experiments were:

$\mathrm{p}_{15}=0.0228$ (coulomb, 1 ) $p_{17}=0.0536$ (coulomb, 2 )

$\mathrm{p}_{16}=0.0302$ (viscous, 1 ) $\mathrm{p}_{19}=0.0500$ (coulomb, 3 )

p20 $=0.0248$ (viscous, 3 ) 